\title{
A variational method in the Sturm-Liouville problem with the Neumann and Dirichlet boundary values
}

\author{
Khapaeva Tatiana Mikhailovna ${ }^{1,2}$ \\ ${ }^{1}$ Department of Calculative Mathematic and Cybernatic, Moscow, Russia \\ ${ }^{2}$ Moscow State Lomonosov's University, MSU, Moscow, Russia
}

\section{Email address:}

tmhapa@yahoo.com

To cite this article:

Khapaeva Tatiana Mikhailovna. A Variational Method in the Sturm-Liouville Problem with the Neumann and Dirichlet Boundary Values. Applied and Computational Mathematics. Vol. 3, No. 4, 2014, pp. 117-120. doi: 10.11648/j.acm.20140304.11

\begin{abstract}
A variational method for calculation of the eigenfunctions and eigenvalues in the Sturm-Liouville problem with the Neumann boundary values is offered. The method is based on a functional, which is introduced in this work. An appropriate numerical algorithm is developed. Calculations for the three potentials are produced: $\sin \left((x-\pi)^{2} / \pi\right), \cos (4 x)$ and the high not isosceles triangle. The method is applied to the Sturm-Liouville problem with the Dirichlet boundary values. Some suppositions about the inverse Sturm-Liouville problem are made.
\end{abstract}

Keywords: Sturm-Liouville Problem, Neumann Boundary Values, Dirichlet Boundary Values, Eigenfunctions, Eigenvalues, Variational Method, Functional, Inversed Sturm-Liuville problem, Algorithm

\section{Introduction}

The aim of this work is calculation of the eigenfunctions (EFs) $y_{1}, \ldots, y_{n}$ and eigenvalues (EVs) $\lambda_{l}, \ldots, \lambda_{n}$ in the Sturm-Liouville (SL) problem in the form of:

$$
y^{\prime \prime}-q(x) y=-\lambda y
$$

with the Neumann boundary values:

$$
y(0)=y^{\prime}(\pi)=0
$$

Several methods of calculation of the EFs and EVs in the Sturm-Liouville problem exist. The bibliography on this question you can find in $[1,2]$. In this work a variational method based on a functional is offered:

$$
\begin{aligned}
& \int_{0}^{\pi}\left[y^{\prime \prime}+(\lambda-q(x)) y\right]^{2} d x+\left|\sqrt{\int_{0}^{\pi} y^{2} d x-1}\right| \\
& +\gamma \sum_{i=2}^{n-1}\left|\int_{0}^{\pi} y_{i} y d x\right|+\beta\left|y^{\prime}(\pi)\right|
\end{aligned}
$$

where $\gamma$ and $\beta$ are chosen sufficiently small.

\section{The Main Point of the Variational Method for the Neumann Boundary Values}

An algorithm is based on the well-known properties of the EFs and EVs:

1. orthogonality of the EFs:

$$
\int_{0}^{\pi} y_{i} y_{j} d x=0, \quad i \neq j
$$

2. monotony and positivity of the EVs:

$$
0<\lambda_{1}<\cdots<\lambda_{n}<\lambda_{n+1}<\ldots
$$

3. normality condition for EFs:

$$
\int_{0}^{\pi} y_{n}^{2} d x=1, \quad \forall n
$$

Let's break the segment $[0, \pi]$ into $k$ pieces. $h=\pi /(k-1)$, $y^{l}=0$ in view of the boundary values.

Let's suppose that the first $n-1$ EFs and EVs are known. Let's base the algorithm construction on the following statement: 
Theorem. Let the functional be defined:

$$
\begin{gathered}
J\left[y^{2}, \ldots, y^{k}, \lambda\right]= \\
h \sum_{j=2}^{k-1}\left[\frac{y^{j-1}-2 y^{j}+y^{j+1}}{h^{2}}+\left(\lambda-q^{j}(x)\right) y^{j}\right]^{2} \\
+\left|\sqrt{h \sum_{j=2}^{k} y^{j 2}}-1\right| \\
+\gamma h \sum_{i=1}^{n-1}\left|\sum_{j=2}^{k} y_{i}^{j} y^{j}\right|+\beta \frac{\left|y^{k}-y^{k-1}\right|}{h}
\end{gathered}
$$

where $\gamma$ and $\beta$ are sufficiently small.

$$
\text { If } \min _{y^{2}, \ldots, y^{k}, \lambda} J\left[y^{2}, \ldots, y^{k}, \lambda\right]=0 \text { subject to } \lambda>\lambda_{\mathrm{n}-1}
$$

and $k \rightarrow \infty$, then the function $\tilde{y}=\left[0, \tilde{y}^{2}, \ldots, \tilde{y}^{k}\right]$ and the value $\tilde{\lambda}$, bringing this minimum, are the $\mathrm{EF}$ and the corresponding EV for the SL problem (1)-(2). At that, these $\mathrm{EF}$ and $\mathrm{EV}$ are different from the previous ones.

Proof. Let the functional (6) reach a zero minimum by $\tilde{y}=\left[0, \tilde{y}^{2}, \ldots, \tilde{y}^{k}\right]$ and $\tilde{\lambda}$. I.e.,

$$
\begin{array}{r}
h \sum_{j=2}^{k-1}\left[\frac{\tilde{y}^{j-1}-2 \tilde{y}^{j}+\tilde{y}^{j+1}}{h^{2}}+\left(\tilde{\lambda}-q^{j}(x)\right) \tilde{y}^{j}\right]^{2} \\
+\left|\sqrt{h \sum_{j=2}^{k} \tilde{y}^{j 2}}-1\right| \\
+\gamma h \sum_{i=1}^{n-1}\left|\sum_{j=1}^{k} y_{i}^{j} \tilde{y}^{j}\right|+\beta \frac{\left|\tilde{y}^{k}-\tilde{y}^{k-1}\right|}{h}=0
\end{array}
$$

Let's direct $k$ to $\infty$. The sums over $k$ in (7) by that transform into the integrals by the trapezium rule with a negligibly small remainder. The expression $\frac{\tilde{y}^{k}-\tilde{y}^{k-1}}{h}$ transforms into the difference statement of the first derivative, and then $\frac{\tilde{y}^{j-1}-2 \tilde{y}^{j}+\tilde{y}^{j+1}}{h^{2}}$ transforms into the difference statement of the second derivative. We derive:

$$
\begin{gathered}
\int_{0}^{\pi}\left[\tilde{y}^{\prime \prime}+(\tilde{\lambda}-q(x)) \tilde{y}\right]^{2} d x+\left|\sqrt{\int_{0}^{\pi} \tilde{y}^{2} d x-1}\right| \\
+\gamma \sum_{i=1}^{n-1}\left|\int_{0}^{\pi} y_{i} \tilde{y} d x\right|+\beta\left|\tilde{y}^{\prime}(\pi)\right|=0
\end{gathered}
$$

The expression under the modulus is the normality condition for EFs, and the integrals under the sum are the scalar product of the EFs (3). Thereby, we derive: $\int_{0}^{\pi}\left[\tilde{y}^{\prime \prime}+(\tilde{\lambda}-q(x)) \tilde{y}\right]^{2} d x=0$ and $\left|\tilde{y}^{\prime}(\pi)\right|=0$, or $\tilde{y}^{\prime \prime}+(\tilde{\lambda}-q(x)) \tilde{y}=0$ and $\tilde{y}^{\prime}(\pi)=0$.

I.e., $\tilde{y}$ meets the SL problem (1)-(2), and $\tilde{y}$ is a EF, and $\tilde{\lambda}$ is a corresponding EV.

Which required to be proved.

The distinction between the derived EF, EV and the previous ones follows from monotony of the EVs (4) and the condition $\tilde{\lambda}>\lambda_{n-1}$.

Thereby, at the first step of our algorithm the following functional

$$
J[y]=\int_{0}^{\pi}\left[y^{\prime \prime}+(\lambda-q(x)) y\right]^{2} d x+\left|\sqrt{\int_{0}^{\pi} y^{2} d x}-1\right|+\left|y^{\prime}(\pi)\right|
$$

is being minimized in $y$ and $\lambda$ under the condition $\lambda>0$. As a result we derive a EF $y_{l}$ and a corresponding $\mathrm{EV} \lambda_{l}$.

In order to derive the second pair of the EF and EV we'll minimize the functional

$$
J[y]+\left|\int_{0}^{\pi} y_{1} y d x\right|
$$

in $y$ and $\lambda$ under the condition: $\lambda>\lambda_{1}$, etc.

The global minimum search was carried out by the Random search method (the procedure Nminimize of the famous software Wolfram Mathematica).

It will be observed, that not always one can at once find the zero minimum with a sufficient accuracy. In this case one has to move enough far away from the previous $\mathrm{EV}$ and then make sequentially a few steps until a given accuracy is achieved. At that, the step must be sufficiently small.

If the step isn't small enough, you can miss the proper minimum. The step was being selected experimentally. The distance from the previous EV was being selected experimentally too.

The described calculations were carried out for the potential $q(x)=\cos (4 x)$ (Fig. 2) and asymmetrical functions $\sin \left((x-\pi)^{2} / \pi\right)$ (Fig. 1) and a not isosceles triangle, whose altitude is much longer then the base.

The results are tabulated in the end of the article.

In Fig. 3, 4, 5 you can see the first, second and the twentieth EFs for the not isosceles triangle.

In Fig. 6, 7, 8 you can see the first, second and the twentieth EFs for $\sin \left((x-\pi)^{2} / \pi\right)$.

In Fig. 9, 10, 11 you can see the first, second and the twentieth EFs for $\cos (4 x)$.

The interval $[0, \pi]$ was partitioned in 100 pieces $(n=100)$. By such conditions the minimum of the functional is of the order of $10^{-5}$. Unfortunately, author's computational capability allows only first $20 \mathrm{EV}$ and EF.

The following EVs one can easily calculate with the help of an asymptotic formula [2] (Theorem 4.14):

$$
\begin{gathered}
\lambda_{n}=\left(n+\frac{1}{2}\right)^{2}+\frac{1}{\pi} \int_{0}^{\pi} q(t) d t- \\
\frac{1}{\pi} \int_{0}^{\pi} q(t) \cos ((2 n+1) t) d t+O\left(\frac{1}{\pi}\right)
\end{gathered}
$$




\section{Briefly about the Variational Method for the Dirichlet boundary values}

The variational method is useful for the Sturm-Liuville problem with the Dirichlet boundary values. In this case the base functional looks:

$$
\int_{0}^{\pi}\left[y^{\prime \prime}+(\lambda-q(x)) y\right]^{2} d x+\left|\sqrt{\int_{0}^{\pi} y^{2} d x}-1\right|+\sum_{i=2}^{n-1}\left|\int_{0}^{\pi} y_{i} y d x\right|
$$

The algorithm is constructed similarly. On the first step of our algorithm the following functional

$$
J[y]=\int_{0}^{\pi}\left[y^{\prime \prime}+(\lambda-q(x)) y\right]^{2} d x+\left|\sqrt{\int_{0}^{\pi} y^{2} d x}-1\right|
$$

is being minimized in $y$ and $\lambda$ under the condition $\lambda>0$. The successive EF and EV are being calculated consequentially from the previous ones.

\section{Briefly about the Variational Method for the Inverse Sturm-Liuville Problem}

So, the variational method provides the ability to solve the direct Sturm-Liuville problem for the various boundary values.

Let's mention briefly the inversed Sturm-Liuville problem: to restore the potential $q(x)$ by the known EFs and Evs. According to Theorem $P$ from [3], in order to determine a potential identically you must have two sets of Evs for the tasks with the different boundary values. For example, one task with the Dirichlet boundary values and another one with the Neumann boundary values.

Possibly, the variational method can help to solve the inversed Sturm-Liuville problem.

The problem is incorrect and can be solved by the regularization method, which was developed by Tikhonov A. N. [4].

\section{Conclusions}

It is significant, that in particular the asymmetrical potentials are of interest. Symmetrical potentials were considered earlier by different methods.

So, we have obtained not only theoretical computations, but the results of calculations for the concrete functions too.

This work demonstrates a use of a present-day software to obtain series of EFs and Evs.

The variational method can be useful in some problems of mathematical physics, based on the Sturm-Liuville problem. Maybe, not only for direct, but for inversed problems too.

\section{Results}

\begin{tabular}{cccc}
\hline & $\cos (\mathbf{4} \boldsymbol{x})$ & $\boldsymbol{\operatorname { s i n }}\left(\boldsymbol{( \boldsymbol { x } - \boldsymbol { \pi } ) ^ { 2 } / \boldsymbol { \pi } )}\right.$ & triangle \\
\hline$\lambda_{1}$ & 0.547 & 0.855 & 1.534 \\
$\lambda_{2}$ & 2.102 & 3.139 & 10.936 \\
$\lambda_{3}$ & 6.531 & 7.122 & 15.43 \\
$\lambda_{4}$ & 11.391 & 13.163 & 19.947 \\
$\lambda_{5}$ & 18.195 & 21.235 & 28.034 \\
$\lambda_{6}$ & 26.95 & 31.282 & 38.101 \\
$\lambda_{7}$ & 38.146 & 43.325 & 49.993 \\
$\lambda_{8}$ & 51.714 & 57.349 & 63.714 \\
$\lambda_{9}$ & 66.937 & 73.336 & 79.802 \\
$\lambda_{10}$ & 84.155 & 91.268 & 97.824 \\
$\lambda_{11}$ & 103.677 & 111.126 & 117.71 \\
$\lambda_{12}$ & 125.503 & 132.896 & 138.932 \\
$\lambda_{13}$ & 148.728 & 148.728 & 162.786 \\
$\lambda_{14}$ & 174.439 & 182.068 & 188.007 \\
$\lambda_{15}$ & 201.706 & 209.42 & 215.223 \\
$\lambda_{16}$ & 230.988 & 238.578 & 244.455 \\
$\lambda_{17}$ & 261.731 & 269.515 & 275.167 \\
$\lambda_{18}$ & 294.457 & 302.199 & 307.895 \\
$\lambda_{19}$ & 328.689 & 336.597 & 342.129 \\
$\lambda_{20}$ & 364.896 & 372.673 & 377.849 \\
\hline
\end{tabular}

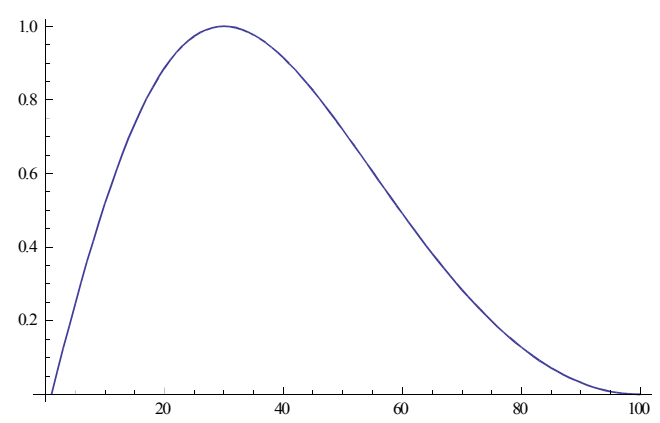

Figure 1. $\sin \left((x-\pi)^{2} / \pi\right)$.

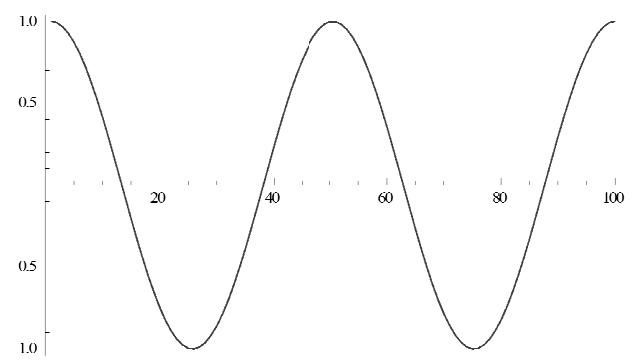

Figure 2. $\cos (4 x)$.

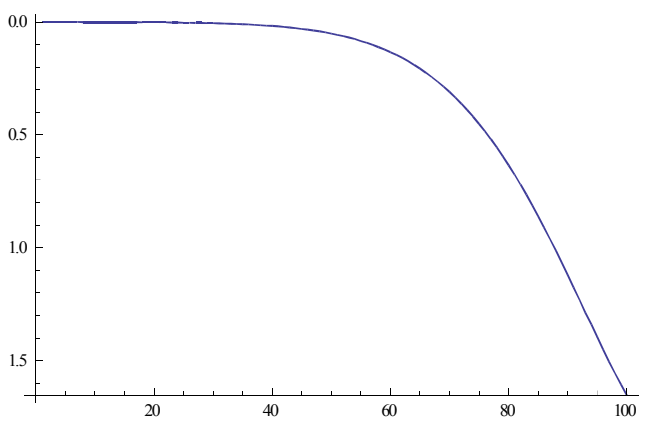

Figure 3. The first EF for the not isosceles triangle. 


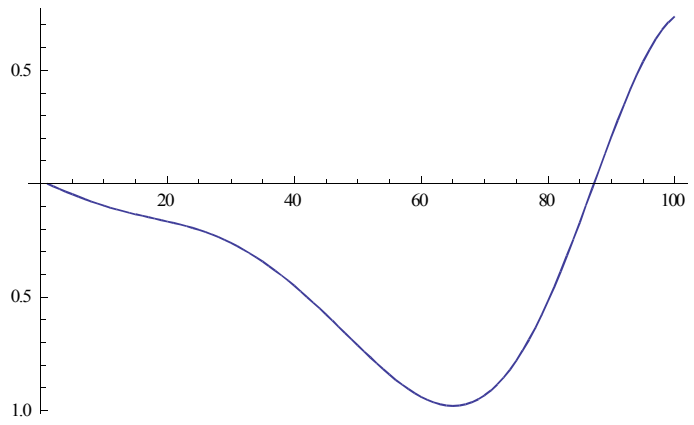

Figure 4. The second EF for the not isosceles triangle.

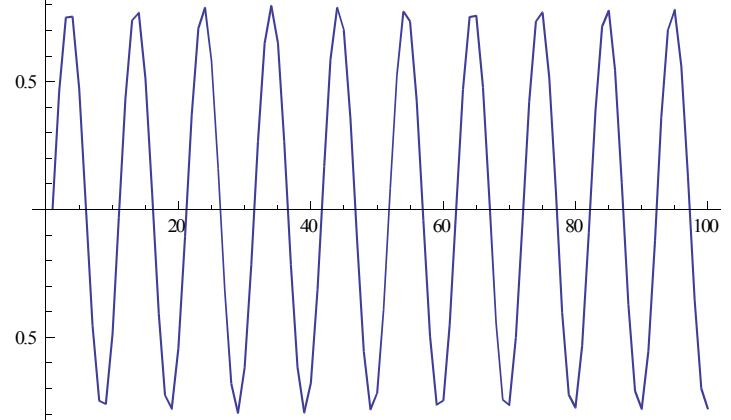

Figure 5. The twentieth EF for the not isosceles triangle.

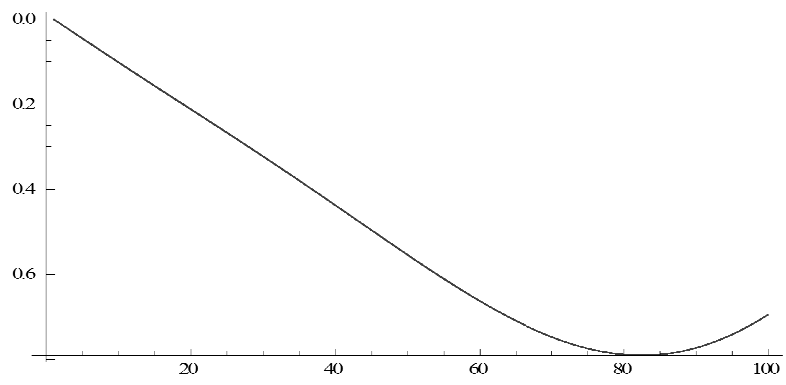

Figure 6. The first $E F$ for $\sin \left((x-\pi)^{2} / \pi\right)$.

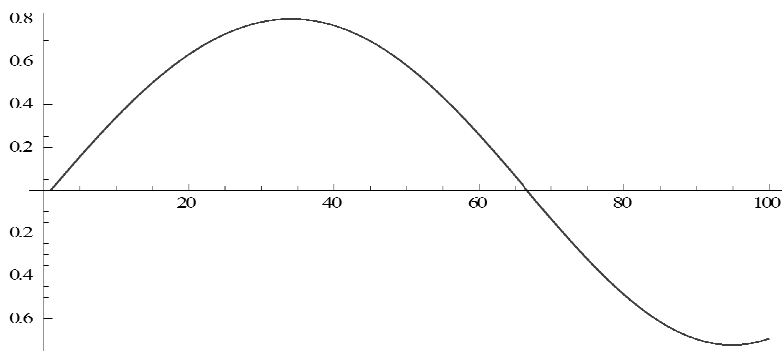

Figure 7. The second $E F$ for $\sin \left((x-\pi)^{2} / \pi\right)$.

\section{References}

[1] L. D. Akulenko and S.V. Nesterov "Jeffektivnyj metod issledovanija kolebanij sushhestvenno neodnorodnyh raspredelennyh system", Prikladnaja matematika i mehanika, vol. $61: 3,1997$, pp. $466-478$

[2] A. Kirsch "An Introduction to the Mathematical Theory of Inverse Problems", 2nd ed, Applied Mathematical Sciences, vol. 120, Springer, 2011

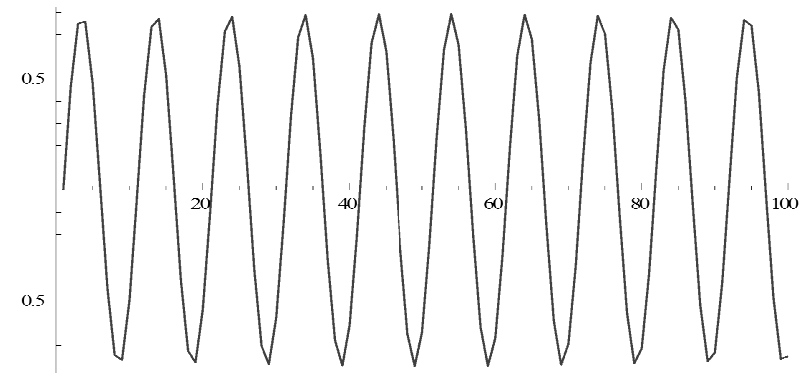

Figure 8. The twentieth EF for $\sin \left((x-\pi)^{2} / \pi\right)$.

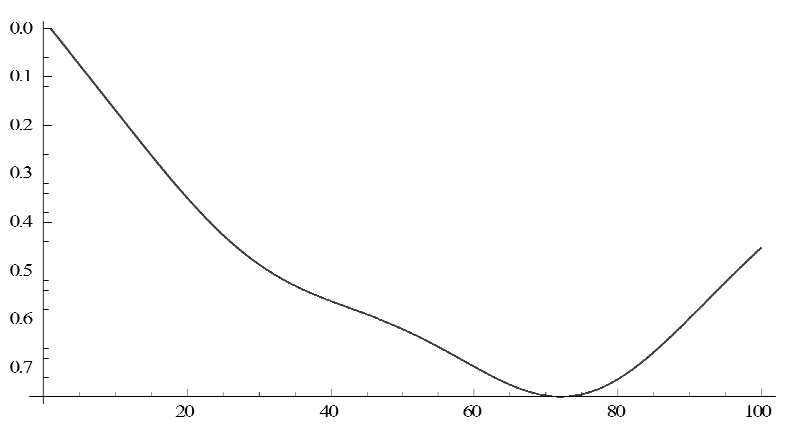

Figure 9. The first EF for $\cos (4 x)$.

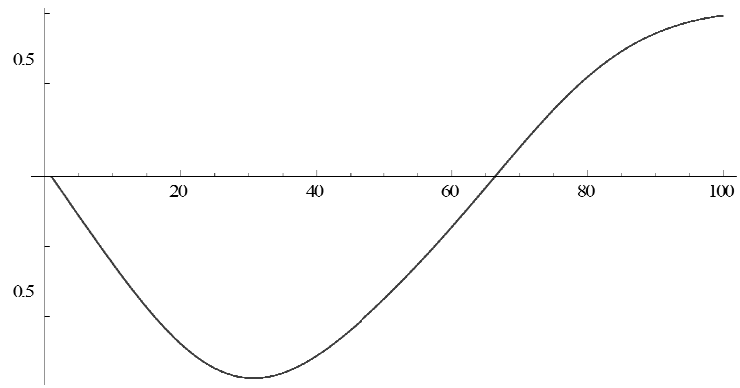

Figure 10. The second $E F$ for $\cos (4 x)$.

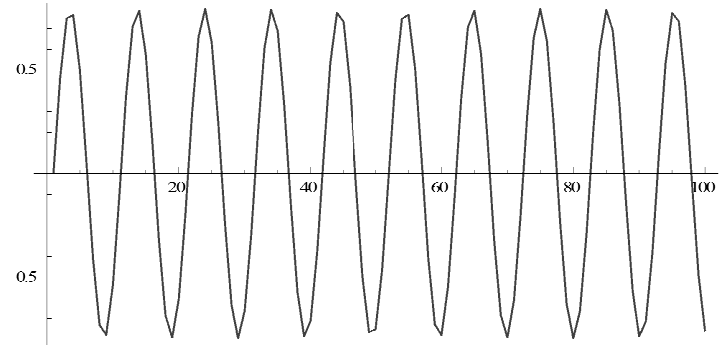

Figure 11. The twentieth EF for $\cos (4 x)$.

[3] Borg G. "Eine Umkehrung der Sturm-Liouvilleschen Eigenwertaufgabe. Bestimmung der Differentialgleichung durch die Eigenwerte", Acta Mathematica, vol. 78, 1946, pp. $1-96$

[4] A. N. Tikhonov and V. Ya. Arsenin, "Methods for Solving Ill-Posed Problems", Nauka, Moscow, 1986 [in Russian] 